\title{
Detection of myocardial ischaemia by transthoracic leads in ambulatory electrocardiographic monitoring
}

\author{
Abstract \\ Objective-To determine the best sites \\ for ambulatory monitoring leads to \\ detect myocardial ischaemia.
}

Patients -50 consecutive patients recovering from myocardial infarction. Six patients were excluded because of unsatisfactory recordings or baseline electrocardiographic abnormalities that influenced the diagnostic accuracy of ST segment depression. In 38 patients important ST segment changes were seen before the study recordings.

Main outcome measure-Reproducibility of detecting the electrocardiographic ST segment changes with 12 bipolar leads alone or in combination.

Results-The highest reproducibility rate was found in infarcts involving both the anterior and inferior left ventricular walls $(80 \%)$. The reproducibility decreased as the extent of ventricular wall involvement decreased and was lowest in inferior infarcts $(31 \%)(p<0.001)$. For large infarcts the detection rate was almost equal for the 12 study leads, whereas disparity between leads increased as the infarct size decreased. The highest overall reproducibility was found in a transthoracic lead (V2, V9R) $(76 \%)$. This lead was significantly better $(p=0.03)$ than lead CM5 (50\%). When the transthoracic lead was combined with an inferior lead, the reproducibility increased $(82 \%)$ and was significantly better than the combination of CM5 and an inferior lead $(58 \%)(p=0.02)$.

Conclusions-Extensive ischaemic electrocardiographic changes are better detected than smaller ones and anterior infarcts better than inferior. A transthoracic lead (V2, V9R) was significantly better than CM5 both alone and when CM5 and the transthoracic lead were combined with an inferior lead.

(Br Heart J 1992;68:286-90)

Holter Laboratory, Hvidovre Hospital, C M Jespersen V Rasmussen

Correspondence to Dr C M Jespersen Østerbrogade 82 DK-2100 Copenhagen $\emptyset$, Denmark.

Accepted for publication 18 November 1991
To assess the optimal sites for ambulatory leads we compared 12 different bipolar leads in patients recovering from acute myocardial infarction.

\section{Patients and methods}

Fifty patients with enzymatically confirmed myocardial infarction were consecutively included in the study. We excluded patients with unstable myocardial diseases, arrhythmias, bundle branch block, electrocardiographic indications of ventricular hypertrophy or strain or both, and patients treated with antiarrhythmic drugs or digoxin because these characteristics interfere with the interpretation of the electrocardiogram. The location of the infarct was identified by serial electrocardiographic recordings, and the infarct location was classified according to the following criteria: changes in leads II, III, aVF as inferior; in V1-V3 (V4) as septal; in V4-V6 as apical; in V6, I, and aVL as lateral; in V1-V6 (I, aVL) as extensive; and when changes were seen in both inferior and anterior walls simultaneously as combined. The type of infarction was classifed on the basis of the presence of abnormal $Q$ waves.

The recordings were carried out before discharge, when the patients were in a stable phase. A standard 12 lead electrocardiogram was recorded immediately before the study recordings and was used as a reference. Twelve bipolar leads were studied (table 1 , fig 1). Four leads (1-4) were sagittally orientated, three (5-7) were placed on the anterior aspect of the thorax, three were oblique leads (8-10), one was transversely orientated (11), and one was a modified inferior lead (12). Leads 11, 12, and 2 corresponded to the orthogonal leads $\mathrm{x}, \mathrm{y}$, and $\mathrm{z}$, respectively, and lead 7 corresponded to CM5.

A segment of each lead comprising at least 200 QRS complexes was stored on a TEAC R-71 tape recorder after amplification. The recordings were analysed by a signal averaging program (HIPEC HA 100). Fifty QRS complexes with a cross correlation factor that was greater than or equal to 0.95 were included in each lead. Low and high filter cut offs were set at 40 and $250 \mathrm{~Hz}$, respectively. The averaged QRS complex of each lead was plotted out on a Sweet-p plotter.

ST segment deflection was measured $80 \mathrm{~ms}$ after the $J$ point and was considered to be significant (positive) if it was $\geq 0.1 \mathrm{mV}$. 
Table 1 Position of negative and positive electrodes in the 12 experimental bipolar leads

\begin{tabular}{|c|c|c|}
\hline & $\begin{array}{l}\text { Negative } \\
\text { electrode }\end{array}$ & $\begin{array}{l}\text { Positive } \\
\text { electrode }\end{array}$ \\
\hline $\begin{array}{l}\text { Sagittal leads: } \\
\quad 1 \\
2 \\
3 \\
4\end{array}$ & $\begin{array}{l}\text { V9 } \\
\text { V3 } \\
\text { V9 } \\
\text { V9 }\end{array}$ & $\begin{array}{l}\text { V2 } \\
\text { V9 } \\
\text { V5 } \\
\text { Xiphoid process }\end{array}$ \\
\hline $\begin{array}{l}\text { Chest leads: } \\
5 \\
6 \\
7\end{array}$ & $\begin{array}{l}\text { Manubrium } \\
\text { Manubrium } \\
\text { Manubrium }\end{array}$ & $\begin{array}{l}\text { V2 } \\
\text { V3 } \\
\text { V5 }\end{array}$ \\
\hline $\begin{array}{r}8 \\
9 \\
10\end{array}$ & $\begin{array}{l}\text { V9R } \\
\text { V9R } \\
\text { V9R }\end{array}$ & $\begin{array}{l}\text { V2 } \\
\text { V3 } \\
\text { V5 }\end{array}$ \\
\hline $\begin{array}{l}\text { Transverse lead: } \\
11 \\
\text { Inferior lead: }\end{array}$ & V6R & V6 \\
\hline 12 & $\begin{array}{l}\text { Superior angle left } \\
\text { scapula }\end{array}$ & $\begin{array}{l}\text { Above the left iliac } \\
\text { crest }\end{array}$ \\
\hline
\end{tabular}

STATISTICAL ANALYSIS

We used the $\chi^{2}$ test with a level of significance set at $\mathrm{p} \leq 0.05$. The difference between proportions (DBP) and the $95 \%$ confidence interval (95\% CI) were calculated by the CIA Statistical package, $B M J$, England, 1990.

\section{Results}

Fifty consecutive patients with acute myocardial infarction were enrolled in the study. Six patients were excluded because of bundle branch block $(n=3)$, atrial fibrillation $(n=1)$, prior enrolment $(n=1)$, and unsatisfactory recording $(n=1)$. The total study population thus consisted of 44 patients. The infarct was inferior in 12, septal in nine, anterior in two, lateral in five, extensive in eight, and combined in eight patients. In $24(55 \%)$ the infarct was a non-Q-wave infarct.

When the study recordings were made $S T$

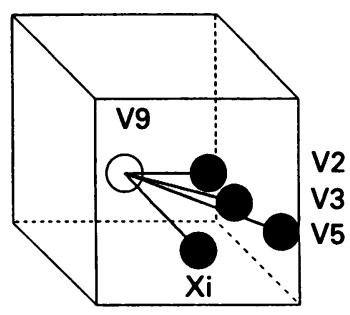

A

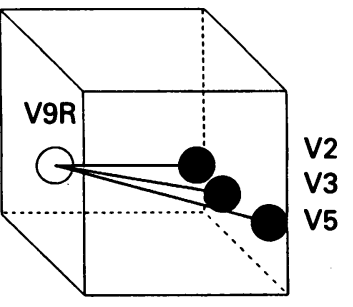

C

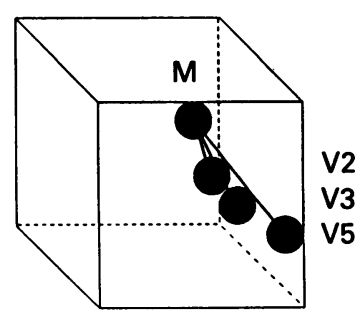

B

LS Caudal
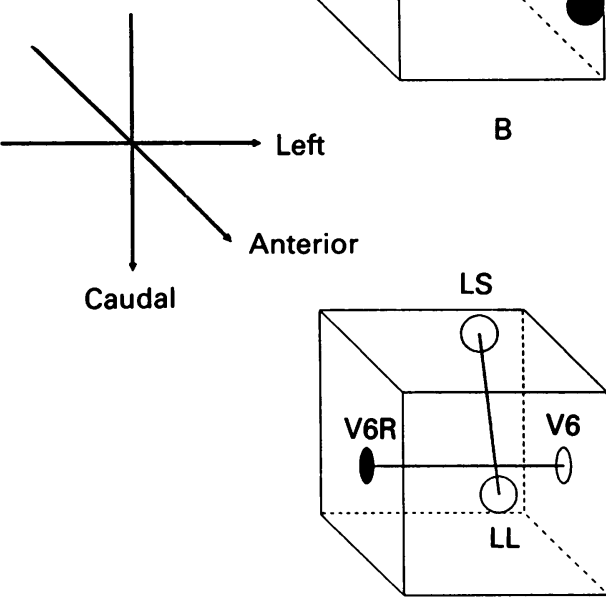

D
Figure 1 Spatial orientation of twelve study leads. (A) sagittal leads (1 to 4 craniocaudal direction); (B) chest leads (5 to 7 from centre to lateral); (C) oblique leads ( 8 to 10 craniocaudal direction); and (D) orthogonal $x$ and $y$ leads. LL, left iliac crest; $L S$, left scapula; $M$, manubrium; $X i$, xiphoid process. segment deflection was seen in the experimental electrocardiographic leads in $38(86 \%)$. In six patients, two with a non-Q wave and four with a $Q$ wave infarct, $S T$ segment deflection had disappeared at the time of recording. Figure 2 shows the number of significant ST segment deflections in the 12 study leads according to infarct location in patients with persisting ST segment deflections in the experimental electrocardiographic leads. The two patients with apical infarcts are not shown. In these patients, however, ST segment deflections were seen in all study leads except in lead 12 in one patient. Figure 2 shows that the total number of positive recordings varied with the location of the infarct. Figure 3 shows overall frequency of positive recordings according to location of the infarct. The detection rate for combined infarcts was significantly higher than for extensive $(p=0.04)$, lateral $(p=0.002)$, septal $(\mathrm{p}<0.001)$, and inferior infarcts $(\mathrm{p}<0.001)$. The detection rate in patients with extensive infarcts was significantly higher than in patients with septal $(p<0.001)$ and inferior infarcts ( $p<0.001)$ but not in patients with lateral infarcts. The detection rate in patients with lateral infarcts was significantly higher than in patients with septal $(p=0.02)$ and inferior infarcts $(p=0.008)$. No significant difference was found between septal and inferior infarcts.

Figure 4 shows the overall detection rates of the study leads. The rate was highest for lead 8 $(74 \%)$. Leads with a detection rate of $50 \%$ or less were significantly inferior to lead $8(50 \%$ : $\mathrm{p}=0.03 ; \mathrm{DBP}=24 \% ; 95 \%$ CI $3 \%$ to $45 \%$ ). In five leads $(1,2,6,8$, and 9$)$ the detection rate exceeded $60 \%$. In all these leads, one electrode was located at the V2 or V3 position and all the leads except one were sagittal or oblique leads. The overall detection rate in lead CM5 (lead 7) was $50 \%$ and detection rates in all leads with one electrode in the V5 position ( 3,7 , and 10) were $50 \%$ or less. The lowest overall detection rate $(39 \%)$ was seen in the inferior lead (12). In patients with inferior infarcts lead 12 was better than CM5, but was surpassed by one sagittal (1) and one oblique lead (9) (fig 2). The combination of leads 8 and 12 gave a detection rate $(82 \%)$ that was better than all other combinations. When lead 7 (CM5) was combined with lead 12 the rate was $58 \%$, which was significantly less than leads 8 and 12 combination $(\mathrm{p}=$ 0.02 ; DBP $=24 \% ; 95 \%$ CI $8 \%$ to $44 \%$ ). When combined with lead 12 , six other leads $(1,2,4,5,6$, and 9$)$ detected more ST segment deflections than the lead 7 and 12 combination, but the differences were not statistically significant. Table 2 shows different combinations of leads 7,8 , and 12 according to small (inferior, septal, lateral) and large infarcts (apical, extensive, combined). For small infarcts the combination of leads 8 and 12 was better than the 7 and 12 combination $(p=0.06$; DBP $29 \%$; $95 \%$ CI $-0 \%$ to $57 \%$ ). In large infarcts, lead $8 \mathrm{missed}$ only one ST segment deflection (fig 2), and no other lead increased the detection rate when combined with lead 8 . Lead 7 detected 12 $(71 \%)$ out of 17 possible deflections in patients with large infarcts. When lead 7 was combined with lead 12 the detection rate increased to 
Figure 2 Number of positive recordings in 12 experimental leads according to infarct location.
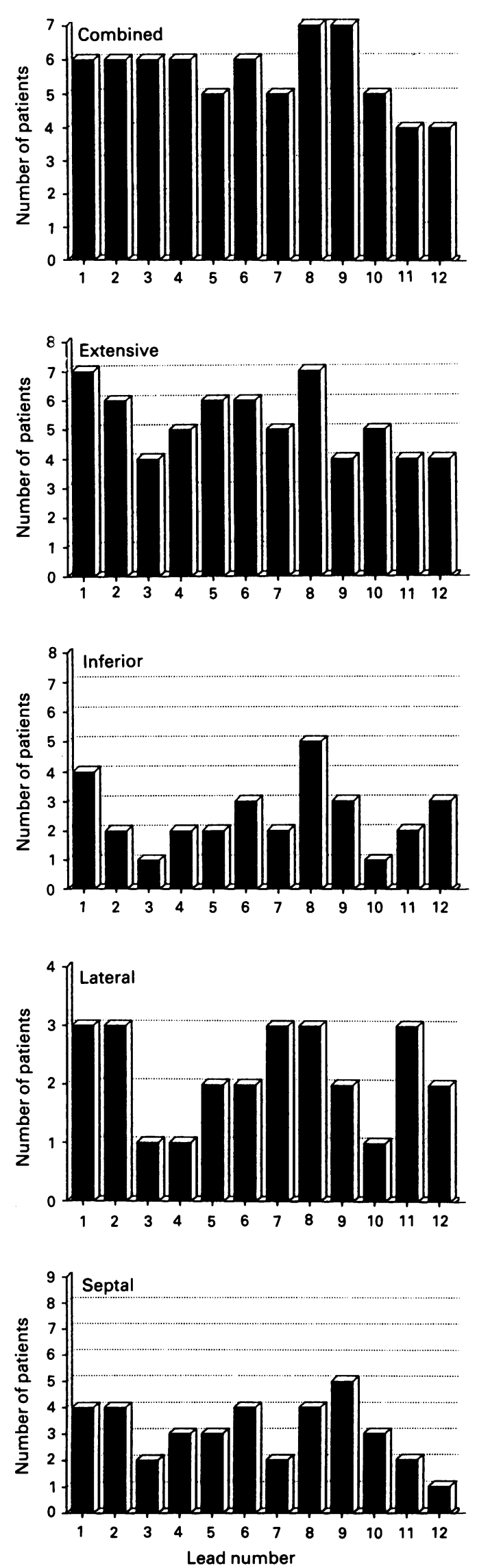

$76 \%$, which was not significant different from the lead 8 and 12 combination.

All combinations detected large infarcts better than small ones. For small infarcts the 8 and 12 combination was best, detecting $71 \%$ of

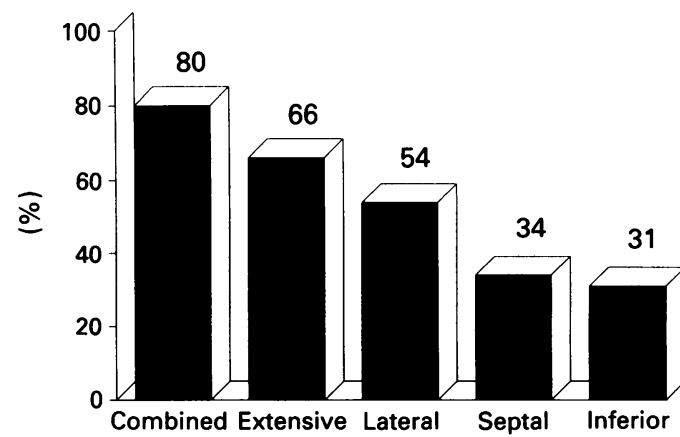

Figure 3 Frequency of significant ST segment deviations in experimental leads according to location of infarct.

small infarcts and $94 \%$ of large ones $(p=0.07$ DBP $0.22 ; 95 \%$ CI 0.00 to $0 \cdot 11$ ). When the 7 and 12 combination was used $71 \%$ of large and $43 \%$ of small infarcts were detected $(p=0.04$; DBP $0.34 ; 95 \%$ CI 0.04 to 0.63 ).

In four patients no significant ST segment changes were seen in the electrocardiogram immediately before the study recordings. In one of these patients study leads $1,2,8,9$ showed a significant displacement. In two patients with serial electrocardiographically verified inferior $Q$ wave infarcts no $S T$ segment changes were seen in the inferior leads in the standard electrocardiogram. In these patients persistent significant ST segment depression was seen in leads V1-V3 and significant changes were found in study leads 1 and 8 and $1,2,4,5$, $7,8,9$, and 11 respectively.

\section{Discussion}

In the present study we used the 12 lead electrocardiogram as a reference for the reproducibility of static postinfarction ST segment changes in 12 experimental leads. As might be expected no single lead reproduced all the changes in configuration shown by a full standard 12 lead electrocardiogram. We found that the reproducibility of regional ST segment changes increased, irrespective of the chosen lead, with increasing myocardial mass involved. The detection rate was highest in infarcts involving both the anterior and inferior left ventricular walls. It decreased as the extent of anterior free wall involvement decreased and was lowest in inferior infarcts. The importance of infarct extension is apparent in fig 2 . For

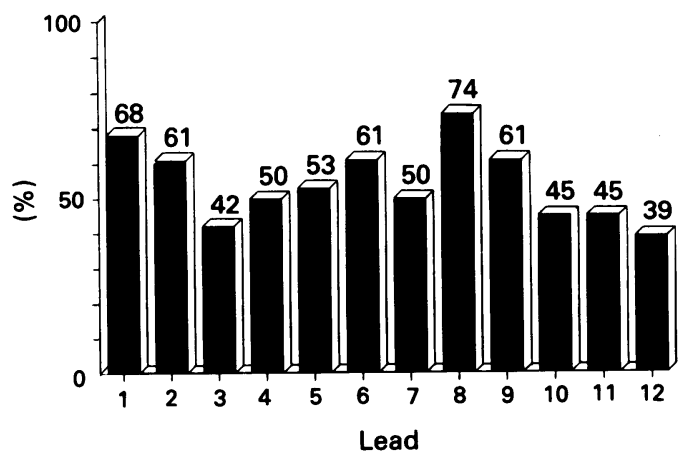

Figure 4 Detection rate (\%) of ST segment deviation in 12 experimental leads. Leads are defined in table 1. 
Table 2 Number of times ST segment deviation was recorded in three selected experimental leads alone or in combination

\begin{tabular}{llcc}
\hline \multicolumn{3}{c}{ Second lead } \\
\cline { 2 - 4 } Main lead & 8 & 7 & 12 \\
\hline All infarcts: & & & \\
$(\mathrm{n}=38)$ & & \\
8 & 28 & 29 & $31^{\star}$ \\
7 & & 19 & $22^{\star}$ \\
12 & & & 15 \\
Small infarcts: & & & \\
$(\mathrm{n}=21)$ & 12 & 13 & $15 \dagger$ \\
8 & & 7 & $9 \dagger$ \\
7 & & & 6 \\
12 & & & \\
Large infarcts: & 16 & 16 & $16 \ddagger$ \\
$(\mathrm{n}=17)$ & & 12 & $13 \ddagger$ \\
8 & & & 9 \\
7 & & & \\
12 & &
\end{tabular}

For definition of infarcts see text.

${ }^{\star} \mathrm{p}=0.02 ; \dagger \mathrm{p}=0.06 ; \ddagger \mathrm{NS}$.

large infarcts the detection rate was almost equal in the 12 study leads, whereas an increased disparity was seen as the infarct size decreased. These results accord with earlier studies. In patients with previous myocardial infarction Castellanet $e t$ al found a significantly lower detection rate for residual inferior ischaemia compared with residual anterior ischaemia. ${ }^{2}$ Fox et al found that the number of leads reflecting myocardial ischaemia increased with the extent and severity of the ischaemia. ${ }^{3}$

Because oblique and sagittal leads "look" through the heart, and chest leads mainly scan the anterior aspects of the heart, oblique and sagittal leads might be better at detecting ischaemia in deeply located myocardial structures. These statements accord with the present study. We found that an oblique lead with one electrode in the V2 position (lead 8) had the highest detection rate, and was more successful than any other lead in patients with combined, extensive, apical, lateral, and inferior infarcts. In ambulatory monitoring CM5 (lead 7) is usually chosen for detection of anterior ischaemia. In the present study, however, many other leads were better than CM5 in patients with combined, extensive, and septal infarcts, and only in patients with apical or lateral infarcts did CM5 reliably detect ST segment deviations. Compared with other chest leads, only those with a more medially placed electrode were better than CM5. Chest leads had low detection rates in patients with inferior infarcts. The inferior lead (12) had a low detection rate in these patients too, detecting less than $50 \%$ of the ST segment changes: only sagittal and oblique leads with one electrode in the V2 position (leads 1 and 8) had a detection rate $\geq 50 \%$ in patients with inferior infarcts.

For ambulatory monitoring two bipolar leads are usually available. Because the combination of CM5 and an inferior like lead is often used in ambulatory monitoring, we compared the 7, and 12 combination with all other possible combinations. In the total cohort with persistent ST segment deflections the detection rate of this 7 and 12 combination was exceeded by seven other combinations all of which included the inferior lead (12) and particularly by the combination of leads 8 and 12, which was significantly better. The main differences were found primarily in patients with small infarcts, in whom the 8 and 12 combination was better $(p=0.06)$ than the CM5 and inferior lead combination. Differences in detection rates for large infarcts between the different leads were small and little extra information was added when two leads were combined.

Our results would be taken to show that two lead ambulatory monitoring primarily detects extensive myocardial ischaemia and is less reliable in detecting ischaemia involving smaller segments. This statement accords with the results obtained in patients with dynamic ST segment changes. In patients recovering from acute myocardial infarction and in patients with stable coronary artery disease, exercise testing with a simultaneous 12 lead electrocardiogram detects significantly more ST segment deviations than does two lead ambulatory monitoring. ${ }^{4-6}$ A positive ambulatory monitoring test, however, identified patients with more extensive electrocardiographic evidence of exercise induced ischaemia, ${ }^{7}$ and was significantly correlated with mortality, reinfarction, and cardiac events. ${ }^{8-10}$ Ambulatory ST segment deflections were primarily seen in patients who also had exercise induced ST segment changes, ${ }^{711} 12$ and Holter monitoring did not add much information on the prevalence of ischaemia in patients with a sufficiently vigorous exercise test. In patients with exercise provoked ischaemia, however, ST segment deflections during ambulatory monitoring identified patients at an increased risk of subsequent cardiac events, ${ }^{89}$ probably because they had extensive ischaemia. Before percutaneous transluminal coronary angioplasty in patients with single vessel coronary disease, ambulatory monitoring showed ST segment deviations in $63 \%$ of patients; $84 \%$ of the deviations were seen in CM5. ${ }^{13}$ During percutaneous transluminal coronary angioplasty the detection rate was $100 \%$ whatever the site of the stenosis.

In four patients no significant ST segment deviations were found in the standard electrocardiogram. In one of them, two sagittal and twooblique leads showed significant deflections, but too few patients were studied for specificity to be calculated.

In ambulatory monitoring, extensive ischaemic electrocardiographic changes are better detected than smaller ones, and anterior changes better than inferior. No single lead or any combination of two leads fully displayed the information given by a standard electrocardiogram. CM5 primarily detected ischaemic changes in the lateral aspects of the left ventricle, whereas septal and inferior changes were partly missed. An oblique bipolar lead was significantly better than CM5, especially in small infarcts. When the oblique lead was combined with an inferior lead the overall detection rate increased and was significantly better than the combination of CM5 and the inferior lead. 
1 Jespersen CM, Rasmussen V. Value of a bipolar modified inferior lead in detection of inferior mycardial ischaemia. Br Heart J 1988;60:287-9.

2 Castellanet MJ, Greenberg PS, Ellestad MH. Comparison of S-T segment changes on exercise testing with angiographic findings in patients with prior myocardial infarction. Am J Cardiol 1978;42:29-35.

3 Fox KM, Deanfield J, Ribero P, England D, Wright C. Projection of ST segment changes on to the front of the chest. Practical implications for exercise testing and ambulatory monitoring. Br Heart J 1982;48:555-9.

4 Gottlieb SO, Gottlieb SH, Achuff SC, et al. Silent ischemia on Holter monitoring predicts mortality in high-risk postinfarction patients. JAMA 1988;259:1030-5.

5 Bonaduce D, Petretta M, Lanzillo T, et al. Prevalence and prognostic significance of silent myocardial ischaemia detected by exercise test and continuous ECG monitoring after acute myocardial infarction. Eur Heart J 1991; 12:186-93.

6 Mulcahy D, Keegan J, Crean P, et al. Silent myocardial ischaemia in chronic stable angina: a study of its frequency
and characteristics in 150 patients. Br Heart $J 1988$; 60:417-23.

7 Currie P, Saltissi S. Transient ischaemia after acute myocardial infarction: relationship to exercise ischaemia. Eur Heart J 1991;12:395-400.
8 Rocco MB, Nabel EG, Campbell S, et al. Prognostic importance of myocardial ischemia detected by le coronary artery disease. Circulation 1988;78:877-84.

9 Deedwania PC, Carbajal EV. Silent ischemia during daily life is an independent predictor of mortality in stable angina. Circulation 1990;81:748-56.

10 Ouyang P, Chandra NC, Gottlieb SO. Frequency and importance of silent myocardial ischemia identified with ambulatory electrocardiographic monitoring in the early in-hospital period after acute myocardial infarction. $\mathrm{Am} \mathrm{J}$ Cardiol 1990;65:267-70.

11 Deedwania PC, Carbajal EV. Exercise test predictors of ambulatory silent ischemia during daily life in stable angina pectoris. Am J Cardiol 1990;66:1151-6.

12 Wilcox I, Freedman SB, Li J, Harris PJ, Kelly DT Comparison of exercise stress testing with ambulatory electrocardiographic monitoring in the detection of myocardial ischemia after unstable angina pectoris. $A m J$ Cardiol 1991;67:89-91.

13 Hoberg E, Schwarz F, Voggenreiter U, Kuebler W. Holter monitoring before, during and after percutaneous transmonitoring before, during and after percutaneous transresolution trend recordings of lead $\mathrm{CM}_{5}$ and $\mathrm{CC}_{5}$ for $\mathrm{ST}$ segment analysis. Am J Cardiol 1987;60:796-800. 